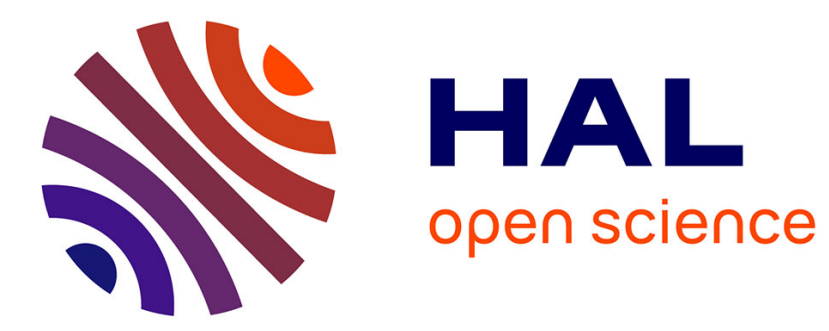

\title{
Recent findings related to immune responses against leptospirosis and novel strategies to prevent infection
}

Frédérique Vernel-Pauillac, Catherine Werts

\section{To cite this version:}

Frédérique Vernel-Pauillac, Catherine Werts. Recent findings related to immune responses against leptospirosis and novel strategies to prevent infection. Microbes and Infection, 2018, 20 (9-10), pp.578588. 10.1016/j.micinf.2018.02.001 . pasteur-02337027

\section{HAL Id: pasteur-02337027}

\section{https://hal-pasteur.archives-ouvertes.fr/pasteur-02337027}

Submitted on 29 Oct 2019

HAL is a multi-disciplinary open access archive for the deposit and dissemination of scientific research documents, whether they are published or not. The documents may come from teaching and research institutions in France or abroad, or from public or private research centers.
L'archive ouverte pluridisciplinaire HAL, est destinée au dépôt et à la diffusion de documents scientifiques de niveau recherche, publiés ou non, émanant des établissements d'enseignement et de recherche français ou étrangers, des laboratoires publics ou privés.

\section{(1) (1) $\$$}

Distributed under a Creative Commons Attribution - NonCommercial - NoDerivatives $\mid 4.0$ 


\title{
Recent findings related to immune responses against leptospirosis and novel strategies to prevent infection
}

\author{
Frédérique Vernel-Pauillac, Catherine Werts* \\ Institut Pasteur, Unité Biologie et Génétique de la Paroi Bactérienne, Groupe Immunité Innée et Leptospires, Paris, France
}

\section{A R T I C L E I N F O}

\section{Article history:}

Received 22 December 2017

Received in revised form

2 February 2018

Accepted 6 February 2018

Available online 13 February 2018

\section{Keywords:}

Leptospira interrogans

Zoonosis

Toll-Like receptors (TLR)

Nod-Like receptors (NLR)

Vaccines

Adjuvants

\begin{abstract}
A B S T R A C T
What are the new approaches and emerging ideas to prevent leptospirosis, a neglected bacterial reemerging zoonotic disease? How do Leptospira interrogans escape the host defenses? We aim here to review and discuss the most recent literature that provides some answers to these questions, in particular data related to a better understanding of adaptive and innate immunity towards leptospires, and design of vaccines. This is an opinion paper, not a comprehensive review. We will try to highlight the new strategies and technologies boosting the search for drugs and vaccines. We will also address the bottlenecks and difficulties impairing the search for efficient vaccines and the many gaps in our knowledge of immunity against leptospirosis. Finally, we aim to delineate how Leptospira spp. escape the innate immune responses of Toll-Like receptors (TLR) and Nod-Like receptors (NLR). The rational use of TLR and NLR agonists as adjuvants could be key to design future vaccines against pathogenic leptospires. (C) 2018 Institut Pasteur. Published by Elsevier Masson SAS. All rights reserved.
\end{abstract}

\section{Introduction: state of the art}

Identified more than 100 years ago, Leptospira interrogans are spirochetes responsible for leptospirosis, also known in its severe form as Weil's disease. For years, leptospirosis has been considered as a dimorphic zoonosis. It was presented in humans, and other incidental hosts, as an acute disease with blood dissemination of the bacteria provoking a potentially fatal, severe disease with multi-organ failure, whereas it was described as an asymptomatic renal colonization in chronic carriers, such as rats. However, recent data suggest that mice, considered as "resistant", may die from an acute disease with uncontrolled leptospires proliferation in blood after intraperitoneal injection with a high inoculum of L. interrogans [1]; conversely, hamsters, used as a model of the acute disease, are chronic carriers of leptospires when they survive experimental infection [2]. Moreover, it has recently been shown that asymptomatic renal carriage of leptospires in humans [3] and pet animals such as dogs [4] has largely been underestimated. Therefore, leptospirosis should be considered as a biphasic disease, starting with an acute phase that is self-resolving in most of the cases, and potentially followed by chronic kidney colonization, depending on the virulence of the strain, the severity of the acute phase and the

\footnotetext{
* Corresponding author

E-mail address: cwerts@pasteur.fr (C. Werts).
}

immune defense of the host. In both acute and chronic manifestations, pathogenic leptospires demonstrate their peculiar ability to overcome the immune system.

Antibiotics efficiently combat leptospirosis, provided they are used early, before leptospires settle in the kidney [1]. The reference treatments are penicillin during the acute phase and streptomycin to cure kidney colonization [5]. However these antibiotic treatments are not generalizable in the long run for animal or human populations living in endemic areas.

In certain situations, prophylactic antibiotic treatments may be appropriate. A treatment with one single dose of $200 \mathrm{mg}$ of doxycyclin can be used to prevent infection with $L$. interrogans in athletes, soldiers and occasional travelers. Azithromycin also seems to be efficient according to our results in mice [1] and in humans (Vinetz, personal communication). Treatments should be carefully evaluated in each animal because the same molecule can present different bioavailability, i.e. prophylaxis with doxycyclin is not efficient in mice because of its reduced life-span compared to humans [1].

Antimicrobial peptides are important components of the eukaryotic innate immune system and promising molecules that have recently been envisioned to counteract leptospires. These small cationic peptides are expressed by virtually all cells, upon activation of pattern recognition receptors (PRR) by microbe associated molecular patterns (MAMP). Besides their potent bactericidal role due to membrane destabilization/depolarization and pore 
formation, they also play a role as cytokines to alert the immune defenses. It has been shown that the rapid expression of antimicrobial defensins participates in promoting acquired immune responses against a potential pathogen [6]. Hopefully, their use could both destroy the leptospires and boost the adaptive immune response (see 3.1 antimicrobial peptides).

Inactivated bacterins (suspensions of killed whole bacteria) remain the only vaccines licensed at the present time. They generate protective antibodies, but are restricted to a given serovar. More than 200 serovars are defined according to the highly immunogenic O-antigen part of the leptospiral lipopolysaccharide (LPS), a T-independent antigen known to generate short-term immunity [7]. Because of these limitations, there is no available universal vaccine that provides cross-protection between different serovars and that is able to block renal colonization. In humans, effective protection often requires repeated immunizations of multivalent vaccines targeting different circulating serovars. This strategy has been efficient to reduce the incidence of human leptospirosis in China and Cuba [8]. This also applies in veterinary use to limit/avoid leptospiral dissemination in livestock.

Side effects due to the local injection of high numbers of bacteria that may trigger adverse inflammatory and allergic reactions are another limitation of such vaccines. Additionally, in some animals, natural infection or vaccination might not systematically trigger a humoral response. For example, in Brazil, multivalent commercial vaccines have been shown inefficient to protect cattle, associated with a complete lack of antibody response [9]. Microscopic agglutination test (MAT) is the gold standard method to determine the serovar specificity and antibody titers in the serum of infected hosts, using a panel of live leptospires representing the prevalent serovars. A recent study highlighted the fact that in a herd of bovines, from which leptospires have been isolated in urine, most of the individuals was tested negative by MAT of their own serovars, including after addition of the local serovar in the MAT panel [10]. These data raise the important issue that individuals with renal colonization and urine excretion of $L$. interrogans may not be detected by MAT, and that other more sensitive tests such as PCR detection of leptospiral DNA should be used. Besides demonstrations that MAT is not sufficiently sensitive and difficult to interpret, these observations also suggests that 1 ) once in their renal niche, leptospires may avoid to trigger the humoral immune response, and 2) in vivo modification of surface proteins of infecting leptospires may be responsible for the inefficiency of the elicited antibodies to recognize in vitro cultured serovars/strains (see 2.2 Posttranslational modifications).

Interestingly, in 2010, studies from the Adler/Murray's group suggested that live attenuated strains with mutation in the LPS biosynthetic pathway [11] protect and induce cross-protection in the hamster model [12]. Although live leptospires, even attenuated, may not be the best option for human vaccines, this study is of great interest since it suggests that the LPS masks the protein antigens and demonstrates that targeting conserved exposed protein antigens is a relevant strategy to get protective and cross reactive vaccines.

Recombinant subunit vaccines are safer than bacterins or live attenuated strains and are largely used to fight infectious diseases. Several recombinant vaccines trials suggest that leptospiral lipoproteins are more or less efficient to protect against lethal challenge but usually do not succeed in sterilizing kidneys [7]. The example of the LipL32 lipoprotein should serve as a caution. This lipoprotein is a very well conserved antigen but proves not to be protective, potentially due to its subcellular localization. Milestones, such as specific antibodies and doing basic tests such as proteinase $K$ "shaving" or immunofluorescence, are essential to insure that the candidate has some chance to be recognized by the host immune antibodies before moving on to animal experiments. However, several lipoproteins that are surface exposed have also proven not to be good vaccines as recombinant proteins. The only convincing example of an efficient subunit vaccine is a recent study using the adhesion protein LigB [13], although a concurrent study challenged the rationale of this success (see 3.3 Subunit vaccines). Hopefully, the availability of large genomic data banks and elaborated structure prediction algorithms, which have recently revolutionized vaccine design, will help our field to develop efficient subunit vaccines against leptospirosis (see 3.2 Reverse and structural vaccinology).

Adjuvants, designed to enhance duration, broadness, and magnitude of antibody production, improve the adaptive immune response to a vaccine antigen. They usually have a dual action: they mechanically favor a slow local delivery of the vaccine and they activate PRR. Hence, they trigger pro-inflammatory pathways, favoring immune cell infiltration, capture, degradation and they promote antigen presentation by dendritic cells (DCs), allowing effector cell maturation. They also reduce the amount of antigen required for immunization, limiting potential local side effects. In contrast to many bacterial infections, leptospirosis and bacterins mostly elicit a T-independent, short-lasting immune response. It indicates that Leptospira spp. by themselves, even alive, are not selfadjuvanting. It is therefore of interest to study how Leptospira spp. escape the innate immune response (See 2.1 Complement escape and 2.3 TLR and NLR species-specific recognition and escape of leptospires) in order to understand how they escape phagocytosis. The final goal of these studies would be to use tailored adjuvants to design leptospiral vaccines (see 3.4 Adjuvants), capable at eliciting a memory response.

Depending on their immune defenses, animals may develop a large spectrum of leptospiral clinical symptoms; from asymptomatic to mild or severe disease, potentially leading to renal colonization, or even to a lethal outcome. Effective anti-leptospiral treatments should target a large panel of hosts. However, different hosts may present species-specificity of recognition of leptospiral MAMPs [14,15] (see 2.3 TLR and NLR species-specific recognition and escape of leptospires). Therefore it is very important to study several animal models and strains to comprehend the immune reactions [16].

Arguably, several recent studies emphasized the role of animals (wild or peridomestic) as reservoirs of leptospires $[4,17,18]$. In particular, the urine is recognized as a favorite route for host shedding of leptospires. As demonstrated in Europe with a wide variety of animals, one study in Tanzania reported that almost 30\% of wild animals were seroreactive against different Leptospira species and serogroups [19], while another study reported renal carriage among $31 \%$ of the mammals tested [20]. Although rats are considered to be the carrier that excretes the highest quantity of leptospires per milliliter of urine, transmission to humans via large mammals, such as cattle, may be more important because of the volume of urine excreted. Correlation between biomes and species/serogroups has been studied in Latin America and Western Indian Ocean islands in order to better understand how the infection is spread. For example in the Seychelles, a recent study showed that rats which are identified as targets for eradication, are not the main reservoir of Leptospira spp. infecting humans. Also, dogs (but not bats) in La Reunion, and ternecs (a small mammal) in Mayotte, have been shown to be reservoir of pathogenic Leptospira spp. contaminating humans [18,17]. Because it seems obvious that serogroups of leptospires are frequently associated with specific animal species, it is very important to target and customize ways to fight the spread of infection depending on the context (epidemiological, geographical, socioeconomic). 


\section{How do Leptospira escape the host defenses?}

\subsection{Complement/phagocytosis escape}

The most important feature explaining the success of pathogenic leptospires to virtually infect all vertebrates (from fishes to mammals) is their ability to escape the complement system, which is the most immediate and efficient innate defense. Pathogenic leptospires use different sophisticated strategies to subvert or inactivate all 3 pathways (classical, alternative and lectins) of the complement cascade. Such mechanisms include covering themselves with host proteases or regulatory complement proteins and secreting proteases. This is not the case of the saprophytic strains that are sensitive to the complement system, and therefore not pathogenic (see the recent review from Barbosa [21]).

Linked to complement evasion, pathogenic leptospires largely escape phagocytosis unless opsonized by specific antibodies [22]. Neutrophils are barely efficient to counteract pathogenic leptospires, but it has been shown ex vivo that L. interrogans are killed by Neutrophil Extracellular Traps (NET) [23]. Neutrophilia is observed in human patients with leptospirosis, but neutrophils are surprisingly not activated [24]. By opposition, in mice, leptospires do not trigger the recruitment of neutrophils in kidneys $[25,15]$, although they recruit macrophages. Interestingly, a recent study showed that in murine macrophages, most of the killing of leptospires occurred through Reactive Oxygen Species (ROS), whereas $L$. interrogans barely stimulated ROS production in the THP-1 human macrophage cell line [26]. This is in line with a previous study from the same group showing cytosolic survival of leptospires in human macrophages, although murine macrophages would kill leptospires, present in vesicles [27]. Ongoing projects in our group aim to understand the underlying hostpathogen mechanisms responsible for the differential human and murine recruitment of macrophages and neutrophils, and their respective killing abilities. This could be instrumental to design strategies that restore the host killing ability against pathogenic Leptospira spp.

\subsection{Post-translational modifications}

An important study came from the group of Nally, using the virulent strain RJ16441 of $L$. interrogans serovar Copenhageni, isolated from the blood of a patient with a severe pulmonary form of leptospirosis. This strain can trigger a lethal infection in the guinea pig model or an asymptomatic infection with renal colonization in the rat model. It was shown that leptospires recovered from livers of moribund guinea pigs presented a modified LPS, with a shortened O-antigen part compared to bacteria cultured in EMJH at $30{ }^{\circ} \mathrm{C}$, or recovered from kidneys of infected asymptomatic rats [28]. In the same line, post-translational modifications (PTM) on LipL32 have been shown to occur in leptospires retrieved from the urine of rats infected with $L$. interrogans serovar Copenhageni. In these bacteria, LipL32 was acetylated or trimethylated on lysines, including those shown to be part of epitopes recognized by antibodies [29]. Therefore, PTM of LPS and LipL32 most probably constitute a leptospiral strategy to escape the immune response. Very interesting studies from the groups of Nally and Caimano addressed more generally the question of in vivo modifications of leptospiral proteins. Dyalysis membrane chambers (DMC) were inoculated with L. interrogans then implanted for 10 days in the rat peritoneal cavity before bacterial recovery. Compared to EMJH cultured bacteria, the authors showed by RNA-Seq that leptospires retrieved from DMC modulated the expression of 166 genes at the mRNA level. Then following mass spectrometry analysis of proteins separated by 2 -
D gel electrophoresis, they showed that LoA22, an outer lipoprotein important for virulence, LipL32, LipL41 and chaperone proteins, are more abundant in vivo. In addition, these proteins were identified to have post-translational modifications, such as phosphorylation or tri-methylations, not observed in vitro when leptospires were grown in EMJH culture medium at $30^{\circ} \mathrm{C}$ or $37^{\circ} \mathrm{C}$ [30]. Therefore, PTM of leptospiral proteins may constitute a hurdle to generate effective vaccines from in vitro cultured bacteria, since candidate proteins will be devoid of these modifications and the antibodies elicited may not recognize the corresponding modified proteins expressed in the host.

\subsection{Toll-Like receptor (TLR) and Nod-Like receptor (NLR) species- specific recognition and escape of leptospires}

Lipopolysaccharide (LPS) is composed of the lipid A, anchored in the outer membrane, linked through the central core to the polysaccharide O-antigen, which extends outside of the bacterium. Lipid A, often referred to as endotoxin, is the agonist of the TLR4 receptor. TLR4 activation leads to a strong inflammatory response and to the recruitment and activation of phagocytes. The favorable outcome of TLR4 activation is the clearance of the bacteria but if the inflammatory response becomes uncontrolled, the host may die of septic shock, consecutive to a cytokine storm. Leptospiral LPS is less endotoxic than LPS from the Gram-negative bacteria such as Escherichia coli or Salmonella spp. L. interrogans' lipid A escapes recognition by human TLR4, although murine TLR4 effectively detects it [14]. Consistent with this result, whilst WT mice are largely asymptomatic, TLR4 deficient mice are highly sensitive to leptospirosis, and may die after intraperitoneal infection with virulent $L$. interrogans. In human, murine, bovine and pig cells, leptospiral LPS is also recognized by TLR2, which is the lipoprotein receptor $[22,31,32]$. Since leptospiral lipid A is not recognized by TLR2, the TLR2 recognition is most probably due to a non-identified lipopeptide tightly attached to the core or $\mathrm{O}$ antigen part of the LPS. In mice, TLR4 and TLR2 activate B cells to produce IgM and protective IgG directed against the LPS. TLR4 and TLR2 are responsible for nitric oxide (NO) production, an efficient bactericidal effector that also exacerbates the renal disease. TLR2 and TLR4 also act together to induce IFN- $\gamma$ [15], which is important to prime the phagocytic activity of macrophages. Of note, lipoproteins are major components of the leptospiral cell wall; more than 140 ORF encode putative lipoproteins, among them the abundant LipL32, LigA, LigB, LipL21 and LoA22. These lipoproteins are thought to be adhesins, binding cells or different components of the extracellular matrix [33]. To date, only LipL32, which is the major lipoprotein specific to pathogenic Leptospira spp., has been shown to activate TLR2. The LipL32/TLR2 complex has been studied in detail by crystallography, showing a $\mathrm{Ca}^{2+}$-binding cluster in LipL32, required for the interaction with TLR2 and subsequent inflammatory signaling. A recent predictive structural study using truncated Lip32 proteins suggests that the complex formation depends on a hydrophobic interaction [34]. Interestingly, one study highlighted that the LPS of L. interrogans serovar Autumnalis strain 56606 only signals through murine TLR4, and not through TLR2 [35]. The authors showed in a TLR4KO mouse model that this strain induces a mild self-resolving acute leptospirosis, affecting liver and lungs with no association to kidney lesions [35]. Together, these results suggest that leptospires, which are potent TLR2 agonists, could use the TLR2 response to subvert and limit the host response. This is under investigation in our laboratory. The recognition of leptospires by TLR involved in nucleic acid recognition (such as TLR9) remains elusive. Ongoing studies in our group also focus on the recognition or escape of leptospires by TLR5 signaling. 
Recent publications show that different leptospiral proteins (hemolysins [36] and Lsa21 [37]) are TLR2 and TLR4 agonists. It is highly probable that these data are misleading since experiments were performed with recombinant proteins produced in $E$. coli, a bacterium expressing one of the most potent LPS. Although increasingly common, using recombinant proteins to study inflammation and innate responses in vitro constitutes a methodological flaw. Indeed, we showed that besides TLR4 activity, the LPS of $E$. coli is naturally rich in contaminants that signals through TLR2, NOD1 and NOD2, and that traces of endotoxin (less than $1 \mathrm{ng} / \mathrm{ml}$ ) synergize with TLR and NLR agonists to induce inflammatory responses [38]. Even if contaminating endotoxins have been largely removed using LPS binding polymixin-based resins, and the endotoxic activity of the recombinant protein evaluated by the limulus amoebocyte lysate (LAL) assay is low, synergies between traces amount of contaminating endotoxin and lipopeptides may occur [38] and account for the observed TLR2/TLR4 activity [39]. To potentially overcome these issues, we tried to express recombinant proteins in an lpxM mutant, or engineered strains of $E$. coli, expressing penta-acylated lipid A, which exhibited only weak TLR4 activity. However our data were not conclusive. The removal of contaminating lipopeptides was not achieved and we found another overlooked issue. We observed that the poly-histidine tag, commonly used to purify recombinant proteins, interfered with the LAL assay. The dose responses were inverted and showed that the more concentrated the protein, the less endotoxin is detected (CW unpublished data about leptospiral HSP70). Since recombinant proteins are often tested for endotoxin contamination at very high concentrations used for cell stimulation $(0,5 \mu \mathrm{g} / \mathrm{ml}$ up to $50 \mu \mathrm{g} / \mathrm{ml})$, the measure of the endotoxin content may be largely underestimated.

The same bias may be highlighted in a study describing LIC11207 [40], potentially involved in delaying neutrophil apoptosis. This study used a recombinant protein, inevitably contaminated with endotoxin, which is well known to exert an anti-apoptotic effect through NF- $\kappa B$ activation. Such issues therefore put the results into question, even though the authors used polymyxin and LPS as controls. In this study and in others, the use of an unrelated protein as a negative control for the phenotype observed can also be misleading, because endotoxin contamination will not be the same depending on the hydrophobicity/polarity of the protein.
Therefore, to avoid these pitfalls, one should absolutely refrain from using those recombinant proteins produced in bacteria to investigate innate immunity. An alternative is to produce leptospiral recombinant proteins in eukaryotic hosts such as Drosophila cells, but not in yeast as the same problem of MAMPs contamination may occur. Nevertheless, recombinant proteins produced in bacteria or yeast have proven very useful in different contexts such as adhesion assays or vaccines. For the latter, the LPS/lipopeptide contamination may provide an interesting adjuvant effect to a given antigen.

IL- $1 \beta$ is a central cytokine in inflammation, important for the expression of other pro-inflammatory cytokines, and whose secretion is tightly controlled by the "inflammasome" [22]. We showed in mice that leptospiral LPS and lipoproteins prime NLRP3 inflammasome and pro-IL-1 $\beta$ mRNA expression. Additionally, the inhibition of the sodium/potassium ( $\mathrm{Na} / \mathrm{K}$ ) pump by glycolipoprotein, an outer membrane component, results in NLRP3 activation and processing of the pro-IL-1 $\beta$ protein to secreted IL-1 $\beta$ [41]. A recent study highlighted a novel role for doxycyclin in inhibiting the IL- $1 \beta$ production in mouse and hamsters cells infected with leptospires [42]. One specific effect of doxycyclin is to restore the $\mathrm{Na} / \mathrm{K}$ pump expression that is inhibited by leptospires, while another role (not specific to leptospires) is to downregulate NLRP3 and IL-1 $\beta$ mRNAs. Altogether, this results in blunting the inflammasome priming and activation and consequently blocks the IL- $1 \beta$ secretion and associated inflammation.

Our recent work showed that $L$. interrogans escape recognition by the cytosolic innate NOD1 and NOD2 receptors, which recognize peptidoglycan fragments called muropeptides [43]. Indeed, we showed that these receptors are not important for the clearance of L. interrogans in mice. This was unexpected since all invasive bacteria, either extracellular or intracellular, are sensed by the NOD receptors. We found that the LipL21 lipoprotein binds to the peptidoglycan and impairs its degradation into muropeptides, which therefore cannot signal through NOD1 and NOD2 (Fig. 1). This protective role of LipL21 seems to be unique to Leptospira spp., since comparison of sequences did not show homology of LipL21 with any other bacterial protein [43]. In addition, we also found a peculiarity of the leptospiral PG composition that influences the species recognition by the NOD1 receptor. Once digested by

\section{$\begin{array}{lll}\text { L. interrogans } & \begin{array}{c}\text { Peptidoglycan (PG) } \\ \text { purification method }\end{array} & \text { Host recognition }\end{array}$}

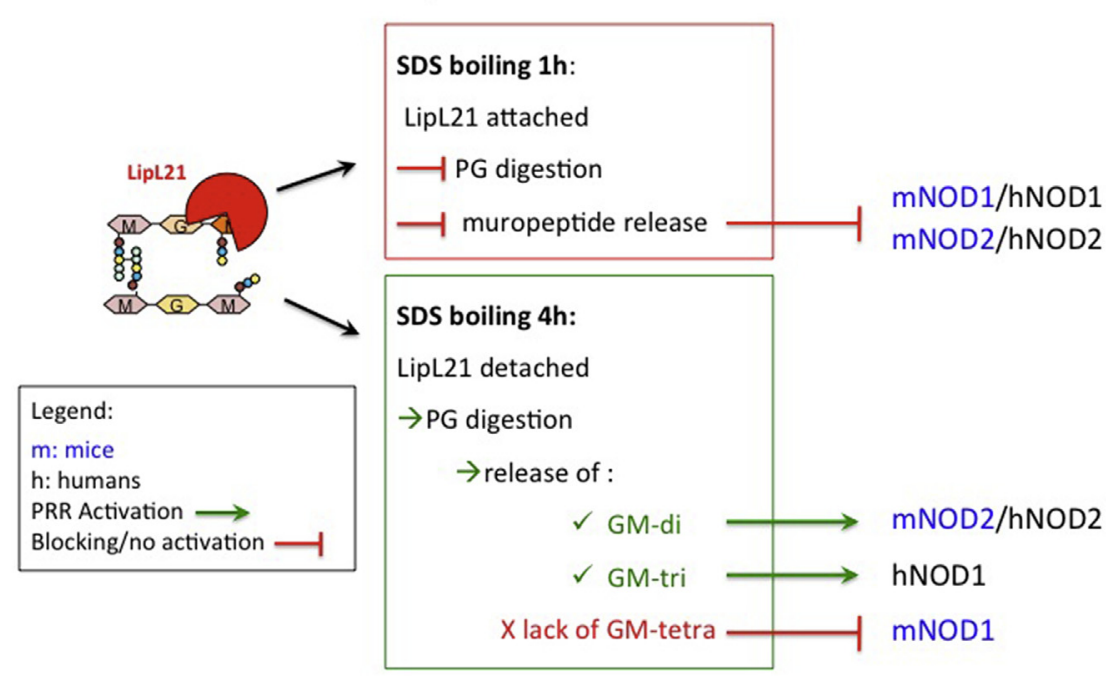

Fig. 1. LipL21 blocks peptidoglycan recognition through the NOD1 and NOD2 receptors. 
hydrolases, the leptospiral PG is almost devoid of GMtetraDAP, a major muropeptide component of the E. coli's PG, which is the preferential agonist of the murine (m)NOD1. In contrast, we found that leptospiral PG is well recognized by the human (h)NOD1 since the major muropeptide is the GM-triDAP, the preferential hNOD1 agonist. Therefore, these results suggest that leptospires counteract both NOD1 and NOD2 receptors. In mice, the presence of LipL21 would only be useful to avoid the NOD2 response since the mNOD1 does not recognize the leptospiral PG. In humans, LipL21 would be more important since the leptospiral PG is perfectly recognized by both NOD1 and NOD2. Our group is now studying the functional consequences of the escape of NOD1 and NOD2 recognition by leptospires in terms of phagocyte functions.

Altogether, the species specificity of TLR4 and NOD1 suggest that a careful study of the recognition of leptospiral cell wall components (LPS, lipoproteins, flagellins, muropeptides ...) by the TLR and NLR of different animals would be instrumental to determine their susceptibility to leptospirosis. These studies may bring interesting insight into the outcome of leptospirosis in different animals, and help to design tailored vaccines, according to the species to be protected.

\subsection{How L. interrogans establish renal chronicity?}

We showed by live imaging using a bioluminescent L. interrogans strain, that following intraperitoneal infection of mice with more than $10^{6}$ bacteria, leptospires replicate in blood, and 1 week post infection disappear from the circulation. They then reappear in the kidneys, where they progressively expand until a stable colonization is established, which occurs 15 days to 1-month post infection [1]. Hamsters have also been shown to be chronic carriers of leptospires when surviving experimental infection $[44,2]$. Recently, the Yoshida group used a hamster model of intradermal inoculation to track post-mortem, after laparotomy, the Murray's LuxCDABE-luminescent $L$. interrogans. Of great interest, they showed that leptospires first disseminate in the subcutaneous adipose tissue before disseminating in the blood and colonizing the organs including the kidneys [45]. Eye drop inoculation is an interesting route of infection since it mimics a natural route of infection and is considered more physiological than intraperitoneal infection, which bypasses the natural defenses of the skin and mucosa. Recently, the Gomes-Solecki group showed in the $\mathrm{C} 3 \mathrm{H} / \mathrm{HeJ}$ TLR4 deficient mouse model that eye inoculation with $10^{8}$ L. interrogans leads to acute leptospirosis with kidney lesions and colonization, although with a delay compared to the intraperitoneal route [46].

An interesting finding recently emerged about the potential role of IL-10, an anti-inflammatory cytokine, in renal colonization. Comparison of cytokines profiles in hamsters and mice infected with leptospires, showed that hamsters, which are sensitive to leptospirosis, express more IL-10 than resistant mice. This augmentation occurs both during the acute phase in the blood and also in kidneys one month post infection $[2,47]$. Interestingly, another study showed that infected IL-10KO mice, in contrast to WT mice, do not exhibit renal colonization. As already stated (in 2.3), IFN- $\gamma$ is a crucial cytokine, decisive for the microbicidal activity of macrophages. IL-10KO mice expressed more IFN- $\gamma$ in the kidneys than WT mice [48]. Up-regulation of IL-10 by leptospires would dampen the IFN- $\gamma$ expression, which would limit the bacterial clearance, hence favoring a chronic infection. Interestingly, it has recently been shown that the IL-10 secretion in the kidneys relies on the TLR2 pathway triggered by leptospires [49]. These data should encourage novel strategies aimed at enhancing phagocytic activity to avoid or limit leptospiral colonization in kidneys.

\subsection{How leptospires maintain chronic infection?}

We strikingly showed a sustained, lifetime asymptomatic renal colonization of $L$. interrogans in mice after intraperitoneal infection [1]. Our results suggest that leptospires are confined in their specific proximal tubule niche and do not expand into new tubules. This could be explained by circulating neutralizing antibodies that could block the exit of leptospires from the tubules and would not allow the expansion of leptospires in the kidney. The proximal tubule is therefore an "immune privileged" site for pathogenic Leptospira spp., but the reason for such an association is unknown. An easy speculation would be that the proximal tubule provides a favorable nutritive environment to leptospires. However, the epithelial layer of the proximal tubule in contact with leptospires is known to express numerous TLR and NLR receptors that will produce antimicrobial peptides upon activation [50]. How leptospires succeed at overcoming this immune defense is a puzzling question. Several ideas are emerging in our field.

Once in their renal niche, we showed in mice that $L$. interrogans were not eradicated by antibiotic treatments, although the same treatments were efficient during the acute phase of infection when leptospires are in the blood. This is in accordance with clinical data showing that antibiotic treatments are effective only if administered early. Once the antibiotic treatment of mice is over, leptospires repopulate their niche, although to a lesser level [1]. We checked that the bacteria were not resistant to the antibiotic, suggesting that these bacteria in tubules are persistent bacteria. As shown in the rat model, these data combined with microscopic studies suggest that leptospires establish dense colonization in the mouse proximal tubules, reminiscent of biofilms. Persisters are dormant bacteria with low metabolism and are supposed to be responsible for biofilm antibiotic resistance. Strategies combining classical antibiotics with drugs able to disperse biofilms should be tested. The use of bioluminescent leptospires to monitor the kidney infection could ease these trials.

Recent studies have highlighted the peculiar role of toxinantitoxins systems [51] and transporters that pump antibiotics out of persister cells [52]. Different toxin/antitoxin modules are present in the genome of leptospires [53-56], and the toxins ChpK and MazF have been shown to be expressed and toxic upon leptospiral infection of human THP-1 macrophages [53]. Future studies of the potential role in virulence and dormancy of these genes upon chronic leptospirosis may be of interest.

\subsection{Influence of sex during the course of infection}

It is established that several criteria influence the degree of severity of leptospirosis; they include the serologic antecedent of a first infection, the infectious dose, the serogroup/serovar of the infecting strain and genetic determinants of the host [57]. In addition, the predisposition related to the sex of individuals is questioned. Indeed, several studies show that leptospirosis more often affects men, with a possible bias consecutive to the practice of activities associated with an increased risk of leptospiral infection [58]. Although prevalence of anti-Leptospira antibodies in both genders was shown to be equivalent regardless of the geographic region influence $[59,60]$, thereby suggesting an equal incidence of infection, thorough epidemiological studies show that males are more often hospitalized and more susceptible to development of severe disease than women [61,62]. Interestingly, an increased male susceptibility was recently reported in a sub-lethal infection model in hamsters, in which males were more prone to develop pulmonary haemorrhage than females [63]. In the latter, an upregulation of nitric oxide (NO) synthase genes in the lungs was associated with the lack of leptospiral detection, and therefore was 
considered as a beneficial factor protecting females against pulmonary damages due to leptospirosis. This is in line with our results showing an upregulation of iNOS in lungs and kidneys of wild-type asymptomatic C56BL/6 mice, whereas mice deficient for both TLR2 and TLR4, highly sensitive to acute leptospirosis, did not upregulate iNOS [15]. Interestingly, a gender difference has been reported regarding the immune responses in mouse airways after LPS sensitization. Males were found to have increased response of proinflammatory cytokines [64]. However the authors did not find any difference in expression of TLR4 in males and females, suggesting that TLR4 did not underlie this gender difference [64]. To our knowledge, a gender bias of susceptibility to acute leptospirosis in mice has never been reported, but most published experiments were performed using females [16]. In our hands, using C56BL/6 mice, we tested both sexes and did not observe a bias in the susceptibility to acute leptospirosis, in terms of lethality, bacterial loads and cytokines production in the liver and the kidneys during the acute phase of infection. Also, using live imaging to check renal colonization, we did not see any difference in the intensity of renal colonization between males and females (unpublished data). Similar results have been reported from the observation of renal colonization in rats from urban slums in Brazil [65]. Therefore, to be truly informative about the influence of sex in the severity of leptospirosis, analyses should include different animal models, target organs, a panel of strains of different serogroups/serovars, and be performed over a longer period of time to test not only the acute phase but also the long term consequences of chronic infection. Considering that pulmonary haemorrhaging has emerged as an important clinical syndrome of human leptospirosis, associated with the highest burden on lethality $[66,61]$, studies to understand the hormonal or genetic component influencing the increased resistance of females could be an important step toward future strategies to limit severe leptospirosis.

\section{New approaches to design drugs and vaccines}

\subsection{Antimicrobial drugs}

Whole blood transcriptomic analyses in leptospirosis patients have recently identified elevated transcripts of effectors involved in the pro-inflammatory signaling pathways and low expression of both the RANTES cytokine and the antimicrobial peptide cathelicidin (LL37) as a correlate to a poor clinical outcome of severe or fatal leptospirosis [67]. Moreover, treatment with synthetic LL37 at the time of infection reduced the bacterial load in blood and rescued $40 \%$ of hamsters from a lethal challenge with $L$. interrogans [67]. This study is important since it may open a new avenue to treat human leptospirosis. The recent finding that LL37 activates NET formation and reduces inflammation [68] may provide a potential mechanism for the observed protection of hamsters at the acute phase of infection, when both the bacterial load and inflammation are reaching their peak. BMAP, a bovine equivalent of LL37, has been shown to bind LPS and to decrease, in vitro, the leptospiral LPS induced signaling [31], which is important to elicit inflammation. Studies still need to determine whether LL37 can be used as a prophylactic treatment and/or, if it is active when administered after experimental infection. However, potentially precluding therapeutic trials, LL37 has recently been shown to be a T-cell auto antigen in psoriasis [69].

Taking advantage of the known anti-bacterial activity of pyrans and heterocycles, pyranoisoxazolones were recently synthesized and shown to constitute novel, safe and specific drugs against Leptospira spp. Interestingly, some derivatives, tested in vitro, were shown to be more efficient than doxycyclin, the reference antibiotic treatment, and active against pathogenic but not saprophytic
Leptospira spp. The most active compound could rescue cyclophosphamide sensitized Balb/c mice from a lethal challenge with L. interrogans serovar Canicola. Of note, this compound also proved efficient to avoid renal colonization. Interestingly, in silico docking experiments suggested that this compound could bind to LipL32, expressed only in pathogenic strains [70], potentially explaining the lack of effect on saprophytic strains. However, further investigation is required to understand if binding to LipL32 can explain the toxicity of these drugs towards pathogenic leptospires. If true, this observation may add one more level of complexity regarding the mysterious role of LipL32 which is not required for growth in vitro, and is perfectly conserved in all pathogenic strains, but dispensable for acute or chronic infection [71].

A novel approach to discover efficient antibacterial agents is based on chemogenomics, and consists at using genetics, structure prediction, virtual screening and 3D structure docking to find drugs and derivatives in ligand libraries targeting several proteins of the same family. A first study deciphered, by substractive genomics, the different families of proteins, conserved between pathogenic strains of leptospires [72]. One of these families corresponds to the Mur ligases, involved in the peptidoglycan biosynthetic pathway. This pathway is essential for prokaryotes but absent in eukaryotes and is therefore a good target for antibacterial drugs. The MurD ligase was further screened against a preexisting ligand library [73]. Using the information obtained in the MurD screen as a lead to model the 3D-structure and activity of ligands and target proteins, the authors found novel ligands of MurC and MurF [74]. Those drugs still await in vitro and in vivo testing to prove their efficiency against leptospires.

\subsection{Reverse and structural vaccinology (RV)}

$\mathrm{RV}$ is a computational biostatistical approach to mine bacterial genomes to search for conserved and protective antigens that could be good vaccine candidates, i.e. genes of secreted or surface exposed proteins or lipoproteins, and predicted to bear B and $\mathrm{T}$ epitopes. This technique has recently been implemented for leptospires (for comprehensive recent reviews see Refs. [75] and [76]). In 2006, the Yang group obtained the first results using bioinformatics and analysis based on the theory of reverse vaccinology. They identified, in the genome of $L$. interrogans serovar Lai strain 56601, 226 genes encoding potentially surface-exposed proteins that might be useful as vaccine candidates [77]. Also, the Haake's group in 2009 used $\beta$-barrel prediction programs to identify 4 novel outer membrane proteins of $L$. interrogans serovar Copenhageni strain Fiocruz L1-130 [78].

Two RV studies that identified conserved vaccine candidates of L. interrogans were recently published $[75,79]$. In the first, the genome of $L$. interrogans serovar Copenhageni was mined for predicted outer membrane proteins and lipoproteins. Among these proteins, the conserved orthologs present in all the pathogenic serovars, harboring outer membrane $\beta$ barrel domains, were further modeled in 3D. Peptides predicted to be surface exposed epitopes of major histocompatibility complex of class II molecules were mapped on the proteins, giving information about their potential immunogenicity $[80,76]$. This study identified 18 outer membrane proteins, including 8 outer membrane lipoproteins, TolC efflux pumps and several TonB-dependent transporters, that bind and transport siderophores, metals, and vitamins [80]. In the second study, the genome of $L$. interrogans serovar Lai strain 56601 was screened with a negative RV approach to exclude the inner membrane and cytosolic proteins, which are usually reliably predicted, in order not to miss potential secreted or outer membrane antigens, which are not always properly annotated. One hundred and twenty-one conserved orthologs from 17 
representative strains of pathogenic Leptospira spp. were identified [79]. Among these potential vaccine candidates against leptospirosis, several are known to be antigenic in human patients, and some orthologs have been shown to be protective against other bacterial infections. Interestingly, 9 outer membrane proteins, including TonB-dependent transporters, constitute promising candidates since they were found in both studies. All these leptospiral vaccine candidates await in vivo testing. A careful analysis of these different RV studies should help to refine and reduce the number of conserved vaccine candidates to test on animals.

The first large scale reverse genetic screen to identify vaccine candidates, that were further tested in vivo, was performed by Murray and Adler, who found 262 genes corresponding to predicted outer membrane proteins or lipoproteins in the genome of L. borgpeterseni serovar Hardjo [44]. Two hundred and thirty eight genes were cloned in $E$. coli and recombinant proteins were purified. Pools of 5 recombinant proteins were mixed with alum as adjuvant and injected twice in hamsters. Six weeks after the first immunization, the hamsters were challenged with a strain of L. borgpeterseni serovar Hardjo that is not lethal but colonizes the kidney. Although most of these proteins elicited specific antibodies and were therefore immunogenic, they did not protect the hamsters from renal colonization [44]. This tremendous work constitutes a reference for further studies, as it provides a consequent list of recombinant proteins that proved to be inefficient as vaccine candidates. It is also a suggestive example of how difficult it may be to generate successful vaccines against leptospires using recombinant whole proteins expressed in E. coli, as leptospires are known to modify their proteins in vivo with post-translational modifications, not performed in E. coli (see 2.2).

\subsection{Subunit vaccines}

The leptospiral immunoglobulin-like (Lig)A and LigB are outer membrane lipoproteins that have been extensively studied as vaccine candidates since they are major antigens in patients with leptospirosis (reviewed in Ref. [7]). They are long modular proteins with 12 repeats of immunoglobulin-like domains, extending from the outer membrane. LigA and LigB are similar in their N-terminal parts but only LigB is conserved among the L. interrogans strains. These proteins are involved in adhesion to extracellular matrix components and have recently been shown to bind host factors that could be important to avoid complement killing during infection. Haake's [81] and McBride's [13] groups recently published about the use of part of these modular proteins as subunit vaccines, in the hamster model of leptospirosis. In Haake's study, although both the recombinant proteins expressing the surface exposed part of LigA (carboxy-terminal part), or the N-terminal part of LigB, were immunogenic, only the construct with LigA was protective against a lethal challenge with $L$. interrogans copenhageni strain Fiocruz L1130 in the hamster model [81]. However, the LigA vaccine failed to achieve kidney sterilization [81]. Surprisingly, in McBride's study, almost the same LigB recombinant protein ( $\mathrm{N}$-terminal region of LigB, without the signal peptide) successfully rescued the hamsters from the lethal challenge and sterilized the kidneys [13]. The protection was not dependent of the dose since same protection was achieved with $100 \mu \mathrm{g}$ and $20 \mu \mathrm{g}$ of vaccine.

Careful comparison of the differences between the immunization protocols of these two studies, using almost the same LigB antigen, same dose and same leptospiral strain for challenge, but giving different outcomes, could give some hints towards obtaining efficient subunit vaccines (Fig. 2). Among the following differences,

\begin{tabular}{|c|c|c|}
\hline & $\begin{array}{c}\text { Haake, } 2017 \\
\text { Evangelista et al., PLoS One. } 2017\end{array}$ & $\begin{array}{c}\text { McBride, } 2017 \\
\text { Conrad et al., PLoS Negl Trop Dis. } 2017\end{array}$ \\
\hline Strain & L. interrogons serovar Copenhageni strain Fiocruz & L. interrogans serovar Copenhageni strain Fiocruz \\
\hline Antigen & $\begin{array}{l}\text { LigB (leptospiral immunoglobulin-like B protein) } \\
\& \text { N-ter Ig-like domains } 0 \text { to } 7 \text { (19-672) } \\
\& \text { Soluble protein } \\
\& \text { Hexa-histidine tag (not removed) }\end{array}$ & $\begin{array}{l}\text { LigB (leptospiral immunoglobulin-like B protein) } \\
\& \text { N-ter Ig-like domains 2-6 (and most of } 1 \text { and 7) (131-645) } \\
\& \text { Insoluble protein then renatured } \\
\& \text { Hexa-histidine tag (not removed) }\end{array}$ \\
\hline Adjuvants & Complete/incomplete Freund adjuvant (CFA/IFA) & Aluminium hydroxyde \\
\hline $\begin{array}{l}\text { Number of } \\
\text { injections }\end{array}$ & 3 injections $(D 0, D+14, D+28)$ & 2 injections $(D 0, D+14)$ \\
\hline Route & Subcutaneous & Intramuscular \\
\hline Challenge & $\begin{array}{l}10000 \text { bacteria ( } 500 \text { fold the } L D 50) \\
\& 1 \text { week after the last injection }(D+35) \\
\& \text { Intraperitoneally }\end{array}$ & $\begin{array}{l}200 \text { bacteria }(10 \text { fold the LDS0) } \\
\& 2 \text { weeks after the last injection }(D+28) \\
\& \text { Intraperitoneally }\end{array}$ \\
\hline Outcome & $\begin{array}{l}\text { No protection } \\
\& \text { No statistical difference with the control group } \\
\& \text { Only } 3 / 8 \text { animals protected } \\
\text { No sterile immunity } \\
\& \text { Only } 1 / 8 \text { animals }\end{array}$ & $\begin{array}{l}\text { Protection } \\
\& \text { Between } 87.5-100 \% \\
\text { Sterile immunity among survivors } \\
\& \text { Between } 87.5-100 \%\end{array}$ \\
\hline
\end{tabular}

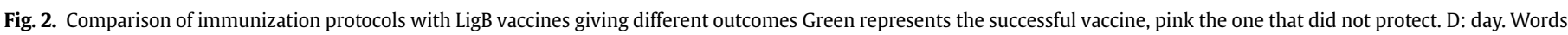
in red correspond to the differences that we hypothesized to be the most important. 
what is the important determinant for the success of vaccination versus (vs) failure $[13,81]$ : production of the recombinant LigB protein (denatured/renatured vs soluble); adjuvants (aluminium hydroxide vs complete/incomplete Freund adjuvant (CFA/IFA)); number of injections and immunization route ( 2 intramuscular vs 3 subcutaneous); number of leptospires to challenge and kill $100 \%$ of non vaccinated hamsters ( 200 vs 10,000 , representing $10-500$ fold the Lethal Dose 50) ? The answer to this question (discussed in Ref. [81]) is of crucial importance to lead future successful trials. In our opinion even though both studies showed $100 \%$ death of animals, Haake's challenge with 10,000 bacteria was more drastic than the 200 bacteria used by the McBride group. The immune system of the hamsters was hence less challenged in McBride's study, potentially explaining a better outcome of this vaccine. Ideally, the repeat of each immunization route and challenge conditions using the other group's construct could be a very potent way to understand the key factors for successful immunization with recombinant proteins.

The Yan group recently published a very interesting study about a multi-epitope chimeric recombinant leptospiral vaccine. This vaccine consists in 4 repeats of a fragment combining 2 previously identified immunodominant $\mathrm{B}$ and $\mathrm{T}$ cell epitopes of each of the 3 major outer membrane proteins OmpL1, LipL32 and LipL21 (conserved and expressed in pathogenic strains) [82]. This vaccine, adjuvanted with alum, and administered subcutaneously, was used in the guinea-pig model of lethal infection with $L$. interrogans strain Lai. It was protective and rescued $80 \%$ of the challenged guinea pigs from death. The vaccine markedly decreased the renal lesions and leptospiral renal colonization was not apparent, as shown by lack of silver staining of leptospires in renal tubules, negative cultures of kidneys and lack of visible leptospires in urine. Interestingly, lymphocytes of guinea pigs stimulated ex vivo with the vaccine showed a Th1 polarized response with IFN- $\gamma$ production. Therefore, this approach is very promising since T cells are recruited in the kidney after leptospiral infection, whilst IFN- $\gamma$ has an important protective role to avoid renal colonization of leptospires (see part 2.4). However, both the potential renal sterilization and the expected protection against different serovars remain to be demonstrated.

\subsection{Adjuvants}

Besides differences in the number and routes of injections in the LigB studies [13,81], one of the major difference was the adjuvant used. Indeed, once good antigen candidates are obtained, the choice of adjuvant is critical.

The Haake group used CFA whereas the McBride group used alum, which is licensed for human vaccines. Alum is one of the most potent adjuvants; it induces a Th2 response by improving the attraction and uptake of antigen by antigen-presenting cells. It activates the NLRP3 inflammasome to produce IL1- $\beta$, leading to neutrophil recruitment at the site of injection and $T$ cells activation [83]. On the other hand, CFA is an emulsion of cell wall components of inactivated dried mycobacteria in mineral oil, usually mixed with a soluble antigen. It has been shown to stimulate NOD2 through MDP. It is however not licensed for humans because of its toxicity, provoking granulomatous inflammatory lesions, and its use in animal experimentation is no longer recommended.

Other PRR agonists may also be good adjuvants. In the hamster, a co-injection of $L$. interrogans serovar Automnalis with the synthetic TLR2 agonist Pam3cysSK4 alleviated the pathology, reduced the bacterial load in organs and improved survival [49]. Interestingly, the authors show that this beneficial effect is correlated with an early up-regulation of TLR2 in mice compared to a delayed TLR2 expression in hamsters. However how these results can be reconciled with the IL-10 production induced by leptospires (described above) to dampen the IFN- $\gamma$ (see paragraph 2.4) is unclear. In this case, TLR2 activation has a positive outcome for the host, whereas it was positive for the bacteria when inducing IL-10.

A recent study compared the protection conferred by two different adjuvants added to a multivalent vaccine in the hamster model of leptospirosis. Pools of selected recombinant conserved outer membrane of leptospiral proteins, complemented or not with a recombinant LigA part (C-term portion), were mixed either with alum or Salmonella Flagellin (FliC) [84]. FliC is a potent agonist of the TLR5 receptor and is a successful vaccine adjuvant. Interestingly, whilst both vaccines with recombinant LigA rescued the hamster after a lethal challenge, only the FliC adjuvant significantly decreased renal colonization, although leptospires were still present in the kidney. This approach is promising since renal sterilization is the hurdle of most leptospiral vaccine trials. Ongoing studies in our group are studying leptospiral recognition/escape by TLR5. Preliminary results suggest that pathogenic Leptospira spp. may naturally escape this defense due to the peculiar localization of the endoflagellum. Strategies aimed at restoring the TLR5 defense are therefore also very promising.

\subsection{Oral vaccines}

Oral vaccination presents several practical advantages over parenteral immunization in developing countries and specialized practitioners are not required to administer the injection. Additionally, oral immunization could be very interesting against a zoonosis like leptospirosis since the vaccines can be widely distributed in food pellets. Mucosal immunization may provide a more appropriate immune response than other routes since pathogenic Leptospira spp. use the mucosal route to infect their hosts. The Gomes-Solecki group recently used live bacteria $[85,86]$ to orally deliver leptospiral antigens. In the first study, live E. coli expressing the C-term part of LigA, were administered daily to hamsters by oral gavage for a period of 4 non-consecutive weeks, before lethal challenge with the strain Fiocruz L1-130 of L. interrogans serovar Copenhageni. The LigA construct was modified with the inclusion of a signal peptide of the OspA lipoprotein from Borrelia spp., conferring a lipidated anchor and a proper localization of the LigA protein at the outer surface of $E$. coli. This recombinant $E$. coli strain expressing LigA induced a high and specific antibody response and rescued $40-60 \%$ of the hamsters from death. However, it did not prevent renal colonization in survivors [85]. Nevertheless, this study demonstrated the feasibility of using a whole organism as a carrier of leptospiral proteins expressed at its surface to induce an immune response against leptospires after oral administration. The yeast Saccharomyces boulardi, administered in food pellets, and generally recognized as a safe (GRAS) organism, was recently shown to enhance the antibody and cytokine responses in Balb/C mice after 3 injections of DNA vaccines expressing LigA or LigB fragments [87]. However, no challenge was done with L. interrogans and it is not known whether $S$. boulardi improves protection against live bacteria.

Another study used the probiotic Lactobacillus plantarum, a GRAS organism known for its immunomodulatory properties. As a first step towards the use of $L$. plantarum as an oral vaccine expressing leptospiral epitopes, $\mathrm{C} 3 \mathrm{H} / \mathrm{HeJ}$ mice were orally gavaged for several weeks with the non-recombinant L. plantarum, before intraperitoneal infection with Fiocruz strain L1-130. Interestingly, Lactobacillus treatment alleviated acute leptospirosis and reduced the renal lesions, although it did not prevent renal colonization. The beneficial effect on the kidneys was correlated with less inflammation and higher recruitment of myeloid cells, in particular 
macrophages and neutrophils [86]. This first result is very promising and ongoing studies are testing different leptospiral proteins expressed in L. plantarum (Gomes-Solecki, personal communication).

\section{Conclusions}

A universal leptospirosis vaccine, safe in humans and protective against all pathogenic Leptospira spp., remains a long-range goal, out of immediate reach. Because of the difficulties of accurate diagnosis and risks of chronic infection, different preventatives, such as efficient drugs or vaccines, should be developed in parallel. To mimic the natural infection, these preventatives should be tested using recently developed animal models challenged through physiological routes.

This last decade, very interesting techniques such as reverse/ structural vaccinology and CRISPR/CAS 9 technologies have opened great prospects and should rapidly ease the research in the field of leptospiral vaccines. Moreover, accurate and sensitive qPCR, and the availability of virulent bioluminescent strains to track the presence of leptospires after challenge, should facilitate vaccine screening. Rats and mice are not always the reservoirs involved in human contamination, and local animals have recently being shown to be overlooked reservoirs favoring human leptospirosis. Hence, local campaigns of mass urine sampling of humans and animals should be organized for both epidemiologic and diagnostic purposes. The identification of asymptomatic humans and animals and circulating serovars would provide the rational for the selection of appropriate strains to use as vaccines. This would also be very important to properly target the relevant animal populations to vaccinate.

Nevertheless, several factors constitute a hurdle against development of effective leptospirosis vaccines.

1 Very basic knowledge regarding the antibody kinetics and the duration of protection after experimental infection is not available for most animal models of leptospirosis. It is therefore difficult to determine whether vaccines could confer long-term protection. The research of vaccines against Leptospira spp. also suffers from the lack of correlates of protection (for a comprehensive discussion see Ref. [44]). Indeed, in the case of leptospirosis, the serological response induced by a vaccine is usually not correlated with efficient protection. The increasing trend to limit animal use because of ethical concerns should be seriously taken into account. One can imagine that correlates of protection could be, in the near future, a pre-requisite to obtain ethical authorization to challenge animals after immunization. Therefore, our field should focus on developing other strategies to test vaccine effects, for example, the measurement of dendritic cells responses after in vitro stimulation with the vaccine or bactericidal phagocytic assays with sera obtained after animal immunization.

2 In vivo, modulation of LPS or proteins by post-translation modifications are most probably responsible for the lack of recognition by antibodies elicited after immunization with strains grown in vitro (e.g in EMJH). Ideally, retrieving leptospires from blood during the hematogenous dissemination would be the best option to obtain proteins with their final conformation (with PTM) as a source of antigens. However this is hardly feasible. The new technique to grow leptospires in vivo (such as the DMC device) could be a nice alternative in some large animals. Another amenable source of host-adapted leptospire strains is the urine. Proteomic studies could be performed using leptospires retrieved from animal and human urine to find antigens that are not the target of PTM.
3 The lipidation of spirochetal proteins confers a potent TLR2 activity, due to the triacylated anchor. The borrelial lipoprotein OspA, already successfully used in vaccines, LipL32 and most probably other leptospiral lipoproteins, are TLR2 agonists. TLR2 agonists have been shown to stimulate dendritic cells and confer subsequent protection against bacterial infections [88]. In the case of leptospirosis, a TLR2 agonist has been shown to be an interesting adjuvant (see 3.4), and lipidated LigA, used in the first oral vaccine, provided a good humoral response (see 3.5). Therefore, the incorporation of a lipid moiety in peptide-based vaccines should be a good strategy for leptospiral vaccines.

4 Other factors are overlooked, such as the reason why there is no immune memory, after leptospiral infection or immunization with bacterins. One simple explanation could be that the LPS masks the responses to conserved proteins. However in the serum of patients, antibodies against several conserved proteins such as LipL32 are found. If TLR2 agonists are good adjuvant and promote T cell responses, why don't leptospires, which are very potent TLR2 agonists, trigger immune memory? This is one question currently being addressed by our group.

5 The lack of targeted mutagenesis has hampered the leptospiral field, however, it is now possible to express heterologous proteins in pathogenic strains from a replicating plasmid (pMAORI), which constitutes a major advance [89]. The new era of genome manipulation with the CRISPR/CAS9 system should encourage trials to construct safe live-attenuated strains complemented in essential genes for in vitro culture, as has been done for Salmonella spp. Also, the new pMAORI plasmid allows the expression of heterogeneous proteins in pathogenic species, which could be used to "adjuvant" the leptospires.

In conclusion, to counteract leptospirosis, which is still not yet recognized by the $\mathrm{WHO}$ as a neglected disease, we need to combine several approaches to construct and optimize novel vaccines. The starting point could be existing vaccines, such as the live attenuated LPS mutant and recombinant LigB vaccines. It could be useful to adjuvant these vaccines with TLR/NLR agonists to try to improve their efficiency. A rational exploration of the recognition (or escape) of Leptospira spp. by receptors and components of the immune system is essential. The escape from TLR and NLR receptors should give some information of how to boost the immune system to alleviate the infection and potentially avoid renal colonization. The escape from complement should also give clues to obtain vaccines that are sensitive to complement and properly processed by phagocytes, potentially eliciting effective $\mathrm{B}$ and $\mathrm{T}$ cell memory.

\section{Conflict of interest statement}

The authors declare no conflict of interest.

\section{Acknowledgments}

We thank Ignacio Santecchia and Delphine Bonhomme, for help with the figures and critical reading, and Richard Wheeler for English editing. This work has been funded by Institut Pasteur.

\section{References}

[1] Ratet G, Veyrier FJ, Fanton d'Andon M, Kammerscheit X, Nicola MA Picardeau M, et al. Live imaging of bioluminescent Leptospira interrogans in mice reveals renal colonization as a stealth escape from the blood defenses and antibiotics. PLoS Negl Trop Dis 2014;8:e3359.

[2] Matsui M, Roche L, Geroult S, Soupe-Gilbert ME, Monchy D, Huerre M, et al Cytokine and chemokine expression in kidneys during chronic leptospirosis in reservoir and susceptible animal models. PLoS One 2016;11:e0156084. 
[3] Ganoza CA, Matthias MA, Saito M, Cespedes M, Gotuzzo E, Vinetz JM Asymptomatic renal colonization of humans in the peruvian Amazon by Leptospira. PLoS Negl Trop Dis 2010;4:e612.

[4] Sant'anna R, Vieira AS, Grapiglia J, Lilenbaum W. High number of asymptomatic dogs as leptospiral carriers in an endemic area indicates a serious public health concern. Epidemiol Infect 2017;145:1852-4.

[5] Martins G, Lilenbaum W. Control of bovine leptospirosis: aspects for consideration in a tropical environment. Res Vet Sci 2017;112:156-60.

[6] Selsted ME, Ouellette AJ. Mammalian defensins in the antimicrobial immune response. Nat Immunol 2005;6:551-7.

[7] Adler B. Vaccines against leptospirosis. Curr Top Microbiol Immunol 2015;387:251-72.

[8] Xu Y, Ye Q. Human leptospirosis vaccines in China. Hum Vaccin Immunother 2017;0. https://doi.org/10.1080/21645515.2017.1405884. PubMed PMID: 29148958.

[9] Favero JF, Fritzen A, Lovato L, Martins P, Baldissera MD, Stefani LM, et al. Immune response of a commercial vaccine against Leptospira interrogans: antibodies and cytokine levels. Microb Pathog 2017;114:46-9.

[10] Libonati H, Pinto PS, Lilenbaum W. Seronegativity of bovines face to their own recovered leptospiral isolates. Microb Pathog 2017;108:101-3.

[11] Murray GL, Srikram A, Henry R, Hartskeerl RA, Sermswan RW, Adler B. Mutations affecting Leptospira interrogans lipopolysaccharide attenuate virulence. Mol Microbiol 2010:78:701-9.

[12] Srikram A, Zhang K, Bartpho T, Lo M, Hoke DE, Sermswan RW, et al. Crossprotective immunity against leptospirosis elicited by a live, attenuated lipopolysaccharide mutant. J Infect Dis 2011;203:870-9.

[13] Conrad NL, Cruz McBride FW, Souza JD, Silveira MM, Felix S, Mendonca KS, et al. LigB subunit vaccine confers sterile immunity against challenge in the hamster model of leptospirosis. PLoS Negl Trop Dis 2017;11:e0005441.

[14] Nahori MA, Fournie-Amazouz E, Que-Gewirth NS, Balloy V, Chignard M, Raetz CR, et al. Differential TLR recognition of leptospiral lipid A and lipopolysaccharide in murine and human cells. J Immunol 2005;175:6022-31.

[15] Chassin C, Picardeau M, Goujon JM, Bourhy P, Quellard N, Darche S, et al. TLR4- and TLR2-mediated B cell responses control the clearance of the bacterial pathogen, Leptospira interrogans. J Immunol 2009;183:2669-77.

[16] Gomes-Solecki M, Santecchia I, Werts C. Animal models of leptospirosis: of mice and hamsters. Front Immunol 2017;8:58.

[17] Lagadec E, Gomard Y, Le Minter G, Cordonin C, Cardinale E, Ramasindrazana B, et al. Identification of Tenrec ecaudatus, a wild mammal introduced to Mayotte Island, as a reservoir of the newly identified human pathogenic Leptospira mayottensis. PLoS Negl Trop Dis 2016;10:e0004933.

[18] Guernier V, Lagadec E, Cordonin C, Le Minter G, Gomard Y, Pages F, et al. Human leptospirosis on Reunion Island, Indian Ocean: are rodents the (only) ones to blame? PLoS Negl Trop Dis 2016;10:e0004733.

[19] Muller SK, Assenga JA, Matemba LE, Misinzo G, Kazwala RR. Human leptospirosis in Tanzania: sequencing and phylogenetic analysis confirm that pathogenic Leptospira species circulate among agro-pastoralists living in Katavi-Rukwa ecosystem. BMC Infect Dis 2016;16:273.

[20] Jobbins SE, Alexander KA. Evidence of Leptospira sp. infection among a diversity of african wildlife species: beyond the usual suspects. Trans R Soc Trop Med Hyg 2015;109:349-51.

[21] Fraga TR, Isaac L, Barbosa AS. Complement evasion by pathogenic Leptospira. Front Immunol 2016;7:623.

[22] Werts C. Interaction of Leptospira with the innate immune system. Curr Top Microbiol Immunol 2017. https://doi.org/10.1007/82_2017_46. PubMed PMID: 29038956.

[23] Scharrig E, Carestia A, Ferrer MF, Cedola M, Pretre G, Drut R, et al. Neutrophil extracellular traps are involved in the innate immune response to infection with Leptospira. PLoS Negl Trop Dis 2015;9:e0003927.

[24] Raffray L, Giry C, Vandroux D, Kuli B, Randrianjohany A, Pequin AM, et al. Major neutrophilia observed in acute phase of human leptospirosis is not associated with increased expression of granulocyte cell activation markers PLoS One 2016;11:e0165716.

[25] Chen X, Li SJ, Ojcius DM, Sun AH, Hu WL, Lin X, et al. Mononuclear-macrophages but not neutrophils act as major infiltrating anti-leptospiral phagocytes during leptospirosis. PLoS One 2017;12:e0181014.

[26] Li S, Li P, Zhang L, Hu W, Wang M, Liu Y, et al. The role of reactive oxygen intermediates in the intracellular fate of Leptospira interrogans in the macrophages of different hosts. PLoS One 2017;12:e0178618.

[27] Li S, Ojcius DM, Liao S, Li L, Xue F, Dong H, et al. Replication or death: distinct fates of pathogenic Leptospira strain Lai within macrophages of human or mouse origin. Innate Immun 2010;16:80-92.

[28] Nally JE, Chow E, Fishbein MC, Blanco DR, Lovett MA. Changes in lipopolysaccharide $\mathrm{O}$ antigen distinguish acute versus chronic Leptospira interrogans infections. Infect Immun 2005;73:3251-60.

[29] Witchell TD, Eshghi A, Nally JE, Hof R, Boulanger MJ, Wunder Jr EA, et al. Posttranslational modification of LipL32 during Leptospira interrogans infection. PLoS Negl Trop Dis 2014;8:e3280.

[30] Nally JE, Grassmann AA, Planchon S, Sergeant K, Renaut J, Seshu J, et al. Pathogenic leptospires modulate protein expression and post-translational modifications in response to mammalian host signals. Front Cell Infect Microbiol 2017;7:362.

[31] Guo Y, Ding C, Zhang B, Xu J, Xun M, Xu J. Inhibitory effect of BMAP-28 on leptospiral lipopolysaccharide-induced TLR2-dependent immune response in bovine cells. Jundishapur J Microbiol 2016;9:e33926.
[32] Guo Y, Fukuda T, Nakamura S, Bai L, Xu J, Kuroda K, et al. Interaction between leptospiral lipopolysaccharide and Toll-like receptor 2 in pig fibroblast cell line, and inhibitory effect of antibody against leptospiral lipopolysaccharide on interaction. Asian-Australas J Anim Sci 2015;28:273-9.

[33] Robbins GT, Hahn BL, Evangelista KV, Padmore L, Aranda PS, Coburn J. Evaluation of cell binding activities of Leptospira ECM adhesins. PLoS Negl Trop Dis 2015;9:e0003712.

[34] Hsu SH, Hung CC, Chang MY, Ko YC, Yang HY, Hsu HH, et al. Active components of Leptospira outer membrane protein LipL32 to toll-like receptor 2. Sci Rep 2017;7:8363.

[35] Xia B, Sun L, Fan X, Xiao H, Zhu Y, Qin J, et al. A new model of self-resolving leptospirosis in mice infected with a strain of Leptospira interrogans serovar Autumnalis harboring LPS signaling only through TLR4. Emerg Microbes Infect 2017;6:e36.

[36] Wang H, Wu Y, Ojcius DM, Yang XF, Zhang C, Ding S, et al. Leptospiral hemolysins induce proinflammatory cytokines through Toll-like receptor 2-and 4-mediated JNK and NF-kappaB signaling pathways. PLoS One 2012;7: e42266.

[37] Faisal SM, Varma VP, Subathra M, Azam S, Sunkara AK, Akif M, et al. Leptospira surface adhesin (Lsa21) induces Toll like receptor 2 and 4 mediated inflammatory responses in macrophages. Sci Rep 2016;6:39530.

[38] Fritz JH, Girardin SE, Fitting C, Werts C, Mengin-Lecreulx D, Caroff M, et al. Synergistic stimulation of human monocytes and dendritic cells by Toll-like receptor 4 and NOD1- and NOD2-activating agonists. Eur J Immunol 2005;35:2459-70.

[39] Gao B, Tsan MF. Endotoxin contamination in recombinant human heat shock protein 70 (Hsp70) preparation is responsible for the induction of tumor necrosis factor alpha release by murine macrophages. J Biol Chem 2003;278: 174-9.

[40] Pretre G, Lapponi MJ, Atzingen MV, Schattner M, Nascimento AL, Gomez RM. Characterization of LIC11207, a novel leptospiral protein that is recognized by human convalescent sera and prevents apoptosis of polymorphonuclear leukocytes. Microb Pathog 2013;56:21-8.

[41] Lacroix-Lamande S, d'Andon MF, Michel E, Ratet G, Philpott DJ, Girardin SE, et al. Downregulation of the $\mathrm{Na} / \mathrm{K}$-ATPase pump by leptospiral glycolipoprotein activates the NLRP3 inflammasome. J Immunol 2012;188:2805-14.

[42] Zhang W, Xie X, Wu D, Jin X, Liu R, Hu X, et al. Doxycycline attenuates Leptospira-induced IL-1beta by suppressing NLRP3 Inflammasome priming. Front Immunol 2017;8:857.

[43] Ratet G, Santecchia I, Fanton d'Andon M, Vernel-Pauillac F, Wheeler R, Lenormand P, et al. LipL21 lipoprotein binding to peptidoglycan enables Leptospira interrogans to escape NOD1 and NOD2 recognition. PLoS Pathog 2017; 13:e1006725.

[44] Murray GL, Lo M, Bulach DM, Srikram A, Seemann T, Quinsey NS, et al. Evaluation of 238 antigens of Leptospira borgpetersenii serovar Hardjo for protection against kidney colonisation. Vaccine 2013;31:495-9.

[45] Ozuru R, Saito M, Kanemaru T, Miyahara S, Villanueva SY, Murray GL, et al. Adipose tissue is the first colonization site of Leptospira interrogans in subcutaneously infected hamsters. PLoS One 2017;12:e0172973.

[46] Sullivan JP, Nair N, Potula HH, Gomes-Solecki M. Eyedrop inoculation causes sublethal leptospirosis in mice. Infect Immun 2017;85(4). pii: e01050-e010516.

[47] Matsui M, Roche L, Soupe-Gilbert ME, Hasan M, Monchy D, Goarant C. High level of IL-10 expression in the blood of animal models possibly relates to resistance against leptospirosis. Cytokine 2017;96:144-51.

[48] Devlin AA, Halvorsen PJ, Miller JC, Laster SM. Il-10 deficient mice express IFNgamma mRNA and clear Leptospira interrogans from their kidneys more rapidly than normal C57BL/6 mice. Immunobiology 2017;222:768-77.

[49] Zhang W, Zhang N, Xie X, Guo J, Jin X, Xue F, et al. Toll-Like Receptor 2 agonist Pam3CSK4 alleviates the pathology of leptospirosis in Hamster. Infect Immun 2016;84:3350-7.

[50] Bens M, Vimont S, Ben Mkaddem S, Chassin C, Goujon JM, Balloy V, et al. Flagellin/TLR5 signalling activates renal collecting duct cells and facilitates invasion and cellular translocation of uropathogenic Escherichia coli. Cell Microbiol 2014;16:1503-17.

[51] Chan WT, Espinosa M, Yeo CC. Keeping the Wolves at Bay: antitoxins of prokaryotic type II toxin-antitoxin systems. Front Mol Biosci 2016;3:9.

[52] Gerdes K, Semsey S. Microbiology: pumping persisters. Nature 2016;534: $41-2$.

[53] Komi KK, Ge YM, Xin XY, Ojcius DM, Sun D, Hu WL, et al. ChpK and MazF of the toxin-antitoxin modules are involved in the virulence of Leptospira interrogans during infection. Microbes Infect 2015;17:34-47.

[54] Lopes AP, Lopes LM, Fraga TR, Chura-Chambi RM, Sanson AL, Cheng E, et al. VapC from the leptospiral VapBC toxin-antitoxin module displays ribonuclease activity on the initiator tRNA. PLoS One 2014;9:e101678.

[55] Picardeau M, Ren S, Saint Girons I. Killing effect and antitoxic activity of the Leptospira interrogans toxin-antitoxin system in Escherichia coli. J Bacteriol 2001;183:6494-7.

[56] Zhang YX, Li J, Guo XK, Wu C, Bi B, Ren SX, et al. Characterization of a novel toxin-antitoxin module, VapBC, encoded by Leptospira interrogans chromosome. Cell Res 2004;14:208-16.

[57] Cachay ER, Vinetz JM. A global research agenda for leptospirosis. J Postgrad Med 2005;51:174-8.

[58] Guerra-Silveira F, Abad-Franch F. Sex bias in infectious disease epidemiology: patterns and processes. PLoS One 2013;8:e62390. 
[59] Alvarado-Esquivel C, Sanchez-Anguiano LF, Hernandez-Tinoco J. Seroepidemiology of Leptospira exposure in general population in rural Durango, Mexico. BioMed Res Int 2015;2015:460578.

[60] Ribeiro P, Bhatt N, Ali S, Monteiro V, da Silva E, Balassiano IT, et al. Seroepidemiology of leptospirosis among febrile patients in a rapidly growing suburban slum and a flood-vulnerable rural district in Mozambique, 20122014: implications for the management of fever. Int J Infect Dis 2017;64: $50-7$.

[61] Costa F, Hagan JE, Calcagno J, Kane M, Torgerson P, Martinez-Silveira MS, et al Global morbidity and mortality of leptospirosis: a systematic review. PLoS Negl Trop Dis 2015;9:e0003898.

[62] Biscornet L, Dellagi K, Pages F, Bibi J, de Comarmond J, Melade J, et al. Human leptospirosis in Seychelles: a prospective study confirms the heavy burden of the disease but suggests that rats are not the main reservoir. PLoS Negl Trop Dis 2017;11:e0005831.

[63] Tomizawa R, Sugiyama H, Sato R, Ohnishi M, Koizumi N. Male-specific pulmonary hemorrhage and cytokine gene expression in golden hamster in early-phase Leptospira interrogans serovar Hebdomadis infection. Microb Pathog 2017;111:33-40.

[64] Card JW, Carey MA, Bradbury JA, DeGraff LM, Morgan DL, Moorman MP, et al. Gender differences in murine airway responsiveness and lipopolysaccharideinduced inflammation. J Immunol 2006;177:621-30.

[65] Santos AA, Figueira CP, dos Reis MG, Costa F, Ristow P. Heterogenic colonization patterns by Leptospira interrogans in Rattus norvegicus from urban slums. Braz J Microbiol 2015;46:1161-4.

[66] Torgerson PR, Hagan JE, Costa F, Calcagno J, Kane M, Martinez-Silveira MS, et al. Global burden of leptospirosis: estimated in terms of disability adjusted life years. PLoS Negl Trop Dis 2015;9:e0004122.

[67] Lindow JC, Wunder Jr EA, Popper SJ, Min JN, Mannam P, Srivastava A, et al. Cathelicidin insufficiency in patients with fatal leptospirosis. PLoS Pathog 2016;12:e1005943.

[68] Hosoda H, Nakamura K, Hu Z, Tamura H, Reich J, Kuwahara-Arai K, et al. Antimicrobial cathelicidin peptide LL37 induces NET formation and suppresses the inflammatory response in a mouse septic model. Mol Med Rep 2017; $16: 5618-26$

[69] Lande R, Botti E, Jandus C, Dojcinovic D, Fanelli G, Conrad C, et al. The antimicrobial peptide LL37 is a T-cell autoantigen in psoriasis. Nat Commun 2014;5:5621.

[70] Ilangovan A, Sakthivel P, Sivasankari K, Mercy CS, Natarajaseenivasan K. Discovery of 6,7-dihydro-3H-pyrano[4,3-c]isoxazol-3-ones as a new class of pathogen specific anti-leptospiral agents. Eur J Med Chem 2017;125: 29-40.

[71] Murray GL. The lipoprotein LipL32, an enigma of leptospiral biology. Vet Microbiol 2013:162:305-14.

[72] Amineni U, Pradhan D, Marisetty H. In silico identification of common putative drug targets in Leptospira interrogans. J Chem Biol 2010;3:165-73.

[73] Umamaheswari A, Pradhan D, Hemanthkumar M. Virtual screening for potential inhibitors of homology modeled Leptospira interrogans MurD ligase. J Chem Biol 2010;3:175-87.

[74] Bhattacharjee B, Simon RM, Gangadharaiah C, Karunakar P. Chemogenomics profiling of drug targets of peptidoglycan biosynthesis pathway in Leptospira interrogans by virtual screening approaches. J Microbiol Biotechnol 2013;23: 779-84.

[75] Dellagostin OA, Grassmann AA, Rizzi C, Schuch RA, Jorge S, Oliveira TL, et al. Reverse vaccinology: an approach for identifying leptospiral vaccine candidates. Int J Mol Sci 2017;18(1). pii: E158.

[76] Grassmann AA, Souza JD, McBride AJ. A universal vaccine against leptospirosis: are we going in the right direction? Front Immunol 2017;8:256.

[77] Yang HL, Zhu YZ, Qin JH, He P, Jiang XC, Zhao GP, et al. In silico and microarray-based genomic approaches to identifying potential vaccine candidates against Leptospira interrogans. BMC Genom 2006:7:293.

[78] Pinne M, Haake DA. A comprehensive approach to identification of surfaceexposed, outer membrane-spanning proteins of Leptospira interrogans. PLoS One 2009;4:e6071.

[79] Zeng L, Wang D, Hu N, Zhu Q, Chen K, Dong K, et al. A novel pan-genome reverse vaccinology approach employing a negative-selection strategy for screening surface-exposed antigens against leptospirosis. Front Microbiol 2017;8:396

[80] Grassmann AA, Kremer FS, Dos Santos JC, Souza JD, Pinto LDS, McBride AJA Discovery of novel leptospirosis vaccine candidates using reverse and structural vaccinology. Front Immunol 2017;8:463.

[81] Evangelista KV, Lourdault K, Matsunaga J, Haake DA. Immunoprotective properties of recombinant LigA and LigB in a hamster model of acute leptospirosis. PLoS One 2017;12:e0180004.

[82] Lin X, Xiao G, Luo D, Kong L, Chen X, Sun D, et al. Chimeric epitope vaccine against Leptospira interrogans infection and induced specific immunity in Guinea pigs. BMC Microbiol 2016;16:241.

[83] McKee AS, Marrack P. Old and new adjuvants. Curr Opin Immunol 2017;47: 44-51.

[84] Monaris D, Sbrogio-Almeida ME, Dib CC, Canhamero TA, Souza GO Vasconcellos SA, et al. Protective immunity and reduced renal colonization induced by vaccines containing recombinant Leptospira interrogans outer membrane proteins and flagellin adjuvant. Clin Vaccine Immunol 2015;22: $965-73$.

[85] Lourdault K, Wang LC, Vieira A, Matsunaga J, Melo R, Lewis MS, et al, Ora immunization with Escherichia coli expressing a lipidated form of LigA protects hamsters against challenge with Leptospira interrogans serovar Copenhageni. Infect Immun 2014:82:893-902.

[86] Potula HH, Richer L, Werts C, Gomes-Solecki M. Pre-treatment with Lactobacillus plantarum prevents severe pathogenesis in mice infected with Leptospira interrogans and may be associated with recruitment of myeloid cells. PLoS Negl Trop Dis 2017:11:e0005870.

[87] Silveira MM, Conceicao FR, Mendonca M, Moreira GM, Da Cunha CE Conrad NL, et al. Saccharomyces boulardii improves humoral immune response to DNA vaccines against leptospirosis. J Med Microbiol 2017;66:184-90.

[88] Jackson DC, Lau YF, Le T, Suhrbier A, Deliyannis G, Cheers C, et al. A totally synthetic vaccine of generic structure that targets Toll-like receptor 2 on dendritic cells and promotes antibody or cytotoxic T cell responses. Proc Natl Acad Sci U S A 2004:101:15440-5.

[89] Pappas CJ, Benaroudj N, Picardeau M. A replicative plasmid vector allows efficient complementation of pathogenic Leptospira strains. Appl Environ Microbiol 2015:81:3176-81. 